\title{
Skin Nodules as a First Presentation of Metastatic Invasive Lobular Carcinoma: Case Presentation
}

\author{
Mohsen Samy Barsoum1, Emad Mohsen Samy Barsoum², Hany Samy Attallah ${ }^{3}$, Hany Nader Victor ${ }^{4}$ \\ ${ }^{1}$ National Cancer Institute (NCI), Cairo University, Cairo, Egypt \\ ${ }^{2}$ Barsoum Oncology Center (BOC), Cairo, Egypt \\ ${ }^{3}$ Military Medical Academy (MMA), Cairo, Egypt \\ ${ }^{4}$ Egyptian Fellowship Medical Oncology (EFMO), Barsoum Oncology Center (BOC), Cairo, Egypt \\ Email: hanysamy2006@yahoo.com
}

How to cite this paper: Barsoum, M.S., Barsoum, E.M.S., Attallah, H.S. and Victor, H.N. (2018) Skin Nodules as a First Presentation of Metastatic Invasive Lobular Carcinoma: Case Presentation. Advances in Breast Cancer Research, 7, 130-133. https://doi.org/10.4236/abcr.2018.72009

Received: March 13, 2018

Accepted: April 17, 2018

Published: April 20, 2018

Copyright $\odot 2018$ by authors and Scientific Research Publishing Inc. This work is licensed under the Creative Commons Attribution International License (CC BY 4.0).

http://creativecommons.org/licenses/by/4.0/

\section{c) (i) Open Access}

\begin{abstract}
Primary breast carcinoma is the most common cause of cutaneous metastases in adult females, comprising about one fourth of patients with the disease. Although CM usually manifests as a late event in the course of a known neoplasm, they are rarely the presenting sign of an undiagnosed malignancy. Invasive Lobular carcinoma is known to be more likely to spread to unusual sites.
\end{abstract}

\section{Keywords}

Lobular Carcinoma, Skin Nodule, First Presentation

\section{Introduction}

Cutaneous metastasis (CM) is an uncommon manifestation of non-dermal malignancies. A meta-analysis showed a $5.3 \%$ incidence of skin metastasis to occur with visceral neoplasm at anytime in the course of the disease [1]. Primary breast carcinoma is the most common cause of cutaneous metastases in adult females, comprising about one fourth of patients with the disease [1] [2].

$\mathrm{CM}$ has different clinical morphology, which can mimic benign skin lesions. Although it is considered a sign of advanced disease, they can be the presenting symptom in rare instances [3].

Here we present a case of Metastatic Invasive Lobular carcinoma, with a precedent, over-looked, skin metastases.

\section{Case Report}

A 54 years old female, presented to our center after Right Modified Radical 
Mastectomy. Postoperative pathology showed an invasive lobular carcinoma with stage pT2N3. Hormone receptor was positive, while HER2 was negative. She also reported a 9-month history of progressive appearance of multiple asymptomatic reddish nodular lesions over her back and head and neck, before mastectomy. She had recent additions to or changes in her medications at that time. Physical examination revealed multiple round-oval, skin nodules, with very firm consistency on palpation, scattered on her back, and head and neck regions (Figure 1), along with her mastectomy scar. Chest X-ray and abdominal ultrasonography were remarkable. Bone scan was requested and showed synchronous bone metastases. Letrozole was initiated. Although bony pains showed a marked response, skin lesions kept to progress on Letrozole. Skin biopsy showed invasive lobular carcinoma of mammary origin. Skin lesion showed no hormone receptor staining while Her-2 kept negative as the primary breast lesion. She was shifted to weekly paclitaxel, from which she received 2 cycles now. Skin lesions show a marked regression. The patient is still alive and responding at the time of submission of this article.

\section{Discussion}

CM may present earlier than other metastatic complications, and dermatologist may be the first consulting specialist to encounter the case [4]. Data published show that CM from all carcinomas is rare. Review of the literature indicates that the incidence of $\mathrm{CM}$ for all types of carcinomas ranges from $0.7 \%$ to $10.0 \%$ [5]. Breast cancer is the most common malignancy to be associated with CM [4]. Different authors have reported incidence of CM from breast carcinoma as $23.9 \%-26.5 \%[1][6]$.

Although CM usually manifests as a late event in the course of a known neoplasm, they are rarely the presenting sign of an undiagnosed malignancy. In a retrospective study of 7316 cancer patients, $6.3 \%$ of the patients with breast cancer presented with skin involvement synchronously with diagnosis of the primary tumor. In $3.5 \%$ of patients, skin involvement was the sign of the disease presentation [7].

In another retrospective analysis of 51 patients presented with CM, Sariya et al. found that forty-five (88\%) of CM were seen in patients with known systemic malignancy. CM was the presenting sign in 12\% (6 of 50). Among 6 patients who had a subsequent diagnosis of a primary malignancy, only 1 case $(1.9 \%)$ had a primary breast cancer [3].

The most common morphology of CM is skin nodule/papule. In a recent retrospective review by Mordenti et al. 164 cases of skin metastases from primary breast carcinoma were examined. The most common clinical finding was skin papules and/or nodules were found in $80 \%$ of patients, telangiectatic carcinomas in $11 \%$, erysipeloid carcinomas in $3 \%$, "encuirasse" carcinomas in $3 \%$, alopecia neoplastica in $2 \%$ and a zosteriform type in $0.8 \%$ [8].

Borst et al. compared the pattern of metastases of Invasive Lobular Carcinoma versus Invasive Ductal carcinoma. He found that ILC was more likely to spread 


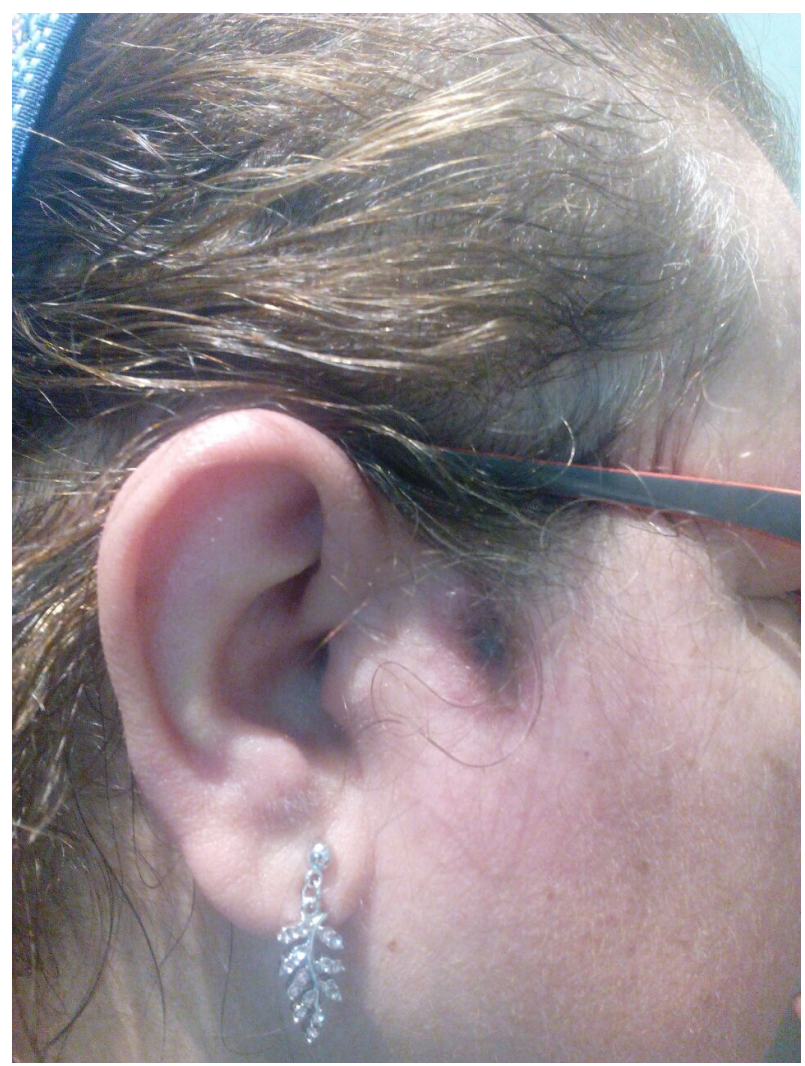

Figure 1. Skin nodule in right zygomatic area.

to unusual sites, like the GI tract, gynecologic organs, peritoneal surface, and retroperitoneum [9]. The etiology of this discrepancy is unknown but it may refer to the loss of expression of the cell-cell adhesion molecule E-cadherin in invasive lobular carcinoma [10]. A debate still exists about the relation between ILC histology and loss of E-cadherin. Singhai et al. found that negative staining of E-cadherin in ILC was specific for the diagnosis of ILC (specificity, 97.7\%; negative predictive value, 96.8\%; 95\% confidence interval, 94.7 - 99.3). However, positive staining did not exclude the diagnosis of ILC [11].

\section{References}

[1] Krathen, R.A., Orengo, I.F. and Rosen, T. (2003) Cutaneous Metastasis: A Meta-Analysis of Data. The Southern Medical Journal, 96, 164-167. https://doi.org/10.1097/01.SMJ.0000053676.73249.E5

[2] International Agency for Research on Cancer (2008) World Cancer Report.

[3] Sariya, D., Ruth, K., Adams-McDonnell, R., Cusack, C., Xu, X., Elenitsas, R., Seykora, J., Pasha, T., Zhang, P., Baldassano, M., Lessin, S.R. and Wu, H. (2007) Clinicopathologic Correlation of Cutaneous Metastases: Experience from a Cancer Center. Archives of Dermatology, 143, 613-620. https://doi.org/10.1001/archderm.143.5.613

[4] Bhattacharyya, A., et al. (2016) Wolf in Sheep's Clothing: A Case of Carcinoma Erysipeloides. Oxford Medical Case Reports, 2016, 97-100. https://doi.org/10.1093/omcr/omw029

[5] Nava, G., et al. (2009) Metastatic Cutaneous Breast Carcinoma: A Case Report and 
Review of the Literature. The Canadian Journal of Plastic Surgery, 17, 25-27. https://doi.org/10.1177/229255030901700105

[6] Spencer, P.S. and Helm, T.N. (1987) Skin Metastases in Cancer Patients. Cutis, 39, 119-121.

[7] Lookingbill, D.P., Spangler, N. and Sexton, F.M. (1990) Skin Involvement as the Presenting Sign of Internal Carcinoma. A Retrospective Study of 7316 Cancer Patients. Journal of the American Academy of Dermatology, 22, 19-26. https://doi.org/10.1016/0190-9622(90)70002-Y

[8] Mordenti, C., Peris, K., Concetta Fargnoli, M., Cerroni, L. and Chimenti, S. (2000) Cutaneous Metastatic Breast Carcinoma. Acta Dermato-Venereologica, 9, 143-148.

[9] Borst, M.J. and Ingold, J.A. (1993) Metastatic Patterns of Invasive Lobular versus Invasive Ductal Carcinoma of the Breast. Surgery, 114, 637-641

[10] Sastre-Garau, X., Jouve, M., Asselain, B., et al. (1996) Infiltrating Lobular Carcinoma of the Breast: Clinicopathologic Analysis of 975 Cases with Reference to Data on Conservative Therapy and Metastatic Patterns. Cancer, 77, 113-120. https://doi.org/10.1002/(SICI)1097-0142(19960101)77:1<113::AID-CNCR19>3.0.C $\underline{\mathrm{O} ; 2-8}$

[11] Singhai, R., Patil, V.W., Jaiswal, S.R., Patil, S.D., Tayade, M.B. and Patil, A.V. (2011) E-Cadherin as a Diagnostic Biomarker in Breast Cancer. The American Journal of the Medical Sciences, 3, 227-233. https://doi.org/10.4297/najms.2011.3227 